\title{
The Dynamic Construal and Generation of Abstract Coherence with the Framework of Cognitive Reference Point, Target Concept Point and the Processing Mental Path
}

Chao Lu

Associate Processor, School of Foreign Studies, University of Science and Technology Beijing, 100083, Beijing, China

Corresponding Author: Chao Lu, E-mail: luchao@ustb.edu.cn

\section{ARTICLE INFORMATION}

Received: November 08, 2020

Accepted: January 14, 2021

Volume: 4

Issue: 1

DOI: $10.32996 /$ ijllt.2021.4.1.1

\section{KEYWORDS}

Embodied Cognition; CRP; RCRP; CTM, Abstracts; local coherence; global coherence; dynamic construal and generation

\section{ABSTRACT}

Langacker's model of Cognitive Reference Point (CRP) and Wang Yin's model of Remedied Cognitive Reference Point (RCRP) could be integrated to form a cognitive trinity to construe and generate the text coherence. This trinity framework is composed of cognitive reference point, target concept point and the processing mental path (CTM) within certain semantic domain. This research tested the efficacy of CTM and found it promotes the dynamic construal and generation of local and global coherence in Abstracts of scientific papers. Activating the related information schema and integrating the mental representation with the semantic information embedded in the Abstract sentences, the three-dimensional CTM establishes a cognitive referential framework for Abstract viewers. This CTM framework assists Abstract viewers in efficiently construing and constructing the local and global coherence of Abstracts.

\section{Introduction}

Scientific papers are the main media to engage the professionals with updated development in scientific and technological fields, and to strengthen academic communication. Abstracts in scientific papers are the essential part where viewers can grasp the research methodology, results and implications quickly for its fully-contained feature of the whole paper. Effective abstracts are highly structured, concise, and coherent and therefore coherence is the key for the concise but clear dissemination of the abstracts (Hesselbach et al.,2012). Coherence is the progression or recurrence of some elements across a span of text. Of the many elements that can recur across text, six more recognizable and concrete elements are the easiest to track, and their coherence is thus the easiest to measure. They are referents, temporality, aspectuality, modality, location and action (Givon, 1993). For text viewers, tracking recurring elements through the text is facilitated by their predictable association with grammatical components. Hence, the distribution of grammatical components in text is a clear methodological shortcut. Explicit grammatical and linguistic signals-syntactic constructions, morphological cues like the use of deictic, anaphoric and cataphoric elements-coupled with conceptual information and thematic information together guide the text processors in the construction of a coherent mental representation of the text. It is a vital cognitive boost and forms a transferring mechanism for placing the clausal information at some coherent location in episodic memory (Gernsbacher,1992). Text representation in episodic memory is represented in part as a network of connected nodes (Weidemann,2019). This network structure displays at least two wellknown features-hierarchy and sequentially. In terms of hierarchy, episodic text representation has at least some depth of hierarchical organization, so that nodes (chunks) are connected both "upward" and "downward" to other hierarchically adjacent nodes. Besides, episodic text representation displays at least some sequential chaining at each hierarchical level, so that nodes are connected to both preceding and following sequentially-adjacent nodes (Norman,2006).

Recently, more researchers have been investigating text coherence from the cognitive perspectives: Givon (1993) stated that coherence is fundamentally not an objective property of the produced text. Rather, that text is a by-product of the mental processes of discourse production and text comprehension, which are the real loci of coherence. van Dijk proposed the concept of "macrostructure", and defined it as "higher-level semantic or conceptual structures that organize the local microstructures of discourse, interaction, and their cognitive processing". Van Dijk claimed that macrostructures could establish the local coherence between sentences and determine the global coherence and structure (1980, pp. 25-28). Beaugrande \& Dressler claimed to

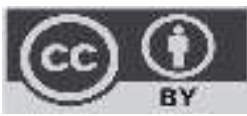

Published by Al-Kindi Center for Research and Development. Copyright (c) the author(s). This open access article is distributed under a Creative Commons Attribution (CC-BY) 4.0 license 
apply semantics to text comprehension, "promoting the related semantic field information in the brain" (1981, p. 33), built the referential relationship among text concepts, and constructed the semantic connection within text. Therefore, the text comprehension becomes the mental processing with unified meaning. Givon pointed out that text coherence is a mental phenomenon, while mental coherence is the prerequisite for construing text coherence (1990:914). Langacker proposed the dynamic text comprehension from the perspective of cognitive grammar (2001, pp. 23-26). He believed that the linear progression of sentences constantly injected new information, and the linear narrative organization could prompt mental processing of text construers. Consequently, text coherence could be restored or generated and whereby coherent text could be integrated in construer's mind. Zhang \&Liu proposed the unification of cognitive schema and psychological thinking into cognitive and psychological chunks and conditions of text coherence could be explored from the perspective of individual cognition (Zhang \& Liu, 2003, pp. 13-14). With neurocognitive platform, Cheng (2011) provided an operational model of cognitive theory for text cohesion and coherence (Cheng Qilong,2011). Wang Yin proposed the cognitive methodology of text analysis (Wang Yin, 2003, pp.83-88; 2011, pp. 6-10), and pointed out that text semantic flow is in a dynamic cognitive process. Therefore, a dynamic framework to analyze the text coherence is more appropriate. This paper aims, from the cognitive perspective, to construe the global and local coherence of abstracts in scientific papers and further generate the local and global coherent schema for the creation of scientific texts.

\section{Literature review}

\subsection{Constructing the cognitive schema for text coherence}

The construal and generation of coherent text lies in the cognitive information framework of text viewers and the corresponding functional cohesive devices of text or sentences. The unified cognitive information is formed from the experience, undergoes cognitive processing and is internalized in people's mind. The cognitive information could be previously accumulated, or newly established information from the current linguistic communication and is the prerequisite for construing and generating text. (Wang Yin, 2005, pp. 16-22) If the lexis from the adjacent sentences or the conceptual information prompted could establish relationship, and the prompting mechanism helps build a unified or accepted cognitive world, the corresponding texts are coherent. Besides, determined by the unified cognitive world information, the linguistic cohesive devices promote the semantic flow.

Scientific texts reflect the scientific research and development. They are re-construe and reconstruct people's common sense. By adopting "descriptive" linguistic feature, scientific texts represent the factual truth and reflect the objective corresponding relationship between language and real world (Koulaidis et al.,2001). In the process of construing scientific texts, the processors of scientific texts integrate the conceptual information, background information, cohesive devices and cognitive schema in their working memory. With the movement of the attention window, the local and global coherence of the text statement is deduced dynamically (Shakiba et al., 2013).

This paper tries to integrate the cognitive theoretical framework of the Cognitive Reference Points(CRP) from Langacker and Remedied Cognitive Reference Points(RCRP) from Wang Yin and aims to construct the three-dimensional cognitive reference model(CTM) to test the coherence realization of scientific texts, which includes referential points and target conceptual points and mental path. Considering the compatibility between CTM mental processing and the mental simulation of language comprehension from the embodied cognitive perspective, and the mental simulation of embodied cognition can achieve better learning and memory effect, this paper attempts to construct a cognitive referential framework CTM, integrate CTM with the embodied cognitive mental simulation of language comprehension, and implement the three dimensional cognitive framework to construe and generate local and global coherence in scientific paper abstract.

\subsection{Mental simulation of language comprehension}

Clark pointed out that the human brain allows mental behavior to take place in a rich, real-world environment (Clark, 1998, pp. 23-26). Therefore, embodied cognitive view of language puts people in a real-world environment, and make mental simulation resonate with previous sensorimotor traces, previous experience and the current situation described by text sentences (Wilson, 2002). The resonation helps comprehend the language describing the non-physical world and realizes, by re-situating, the creative embodied process of language comprehension. Besides, the milestone view that cognition is situational has been supported in a series of researches related to dynamic cognition (Barsalou, 2003, pp. 84-91; Chiel,1997, pp. 553-557). Hence, mental simulation is a new illustration that embodied cognition presents in language comprehension. The core concept is to associate language with sensorimotor memory traces, and construct the foundation for mental simulation. The recognition process of sensorimotor objects is closely related to learners' interactive ways with them (Yang et al.,2011). The brain has limited attention and memory. As a result, the mental simulation that corresponds with neural mechanism and different brain regions

used to recognize different physical objects could maximize the improvement of long-term memory (Nicholas, 2007). Glenberg \& Robertson (1999) found in their research that language recipients, in their language information processing, could acquire 
longer memory duration, higher reading test score, and better learning effect if they correlate the deictic words with context (Glenberg,1999, pp. 1-26; 2011, pp. 1-10). That is, under the conditions of limited cognitive attention and working memory, language comprehension under mental simulation can achieve higher learning effects.

As a new illumination of language comprehension, mental simulation and re-situating highlight the bridging between the conceptual information expressed in the vocabulary and previous sensorimotor memory traces. This new illumination is complementary to the construal of text coherence with Langacker's CRP. That is, using mental simulation, conceptualizer locates cognitive starting points from some cognitive referential(R) words of certain semantic domain, follow certain mental simulation path, track and target the information points of target domain, integrate the mentally unified cognitive world, referential points and the sensory traces prompted by the target points, dynamically construct the cognitive schema based on cognitive reference points, target points and semantic domain and restore or generate the text coherence.

\subsection{Cognitive reference points and text coherence}

Langacker, with the phenomenon of "Nighttime Sky", introduces cognitive reference point (CRP)model. If observers want to locate one star from night starry sky, they (conceptualizers) often need to locate a prominent star (reference point), and then take it as the starting point to locate the target star (target). In this CRP model, D refers to the referential domain of this cognitive processing (Langacker, 2004, pp. 13-20). The dynamic cognitive reference model from Langacker is shown in Fig.1(Li, 2008). The dynamic processing of CRP can be presented as conceptualizer selecting a reference point, in the activated cognitive domain of the brain, following certain mental path, understanding and locating target concept and finally construing and generating the local and global text coherence. However, the ways of coherence vary in actual texts. It is difficult for Langacker's CRP model to fully construe and generate the coherence of realistic text. Therefore, Wang (2011) proposed the remedied cognitive reference point (RCRP) to construe the variable styles in actual texts. The dynamic processing of RCRP is shown in Fig.2(Wang, 2011, pp. 6-10). There are seven connection types of RCRP: hub-and-spoke R-T connection type, nested chain R-t connection type, $R=D$ connection type, $D-T$ diversity type, multiple points referential type, I-He referential type, multiple crossover type. This paper aims to, with hub-and-spoke R-T connection type and nested chain R-T connection type, construe and generate the Abstract coherence in scientific paper.

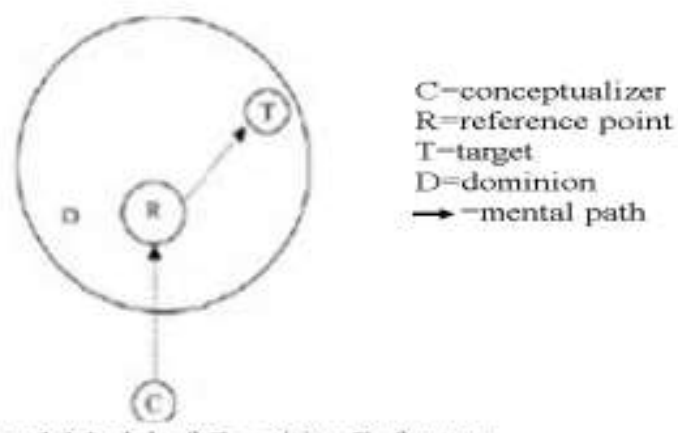

Figure. 1 Model of Cognitive Reference Point (Langacker, 2000:174)

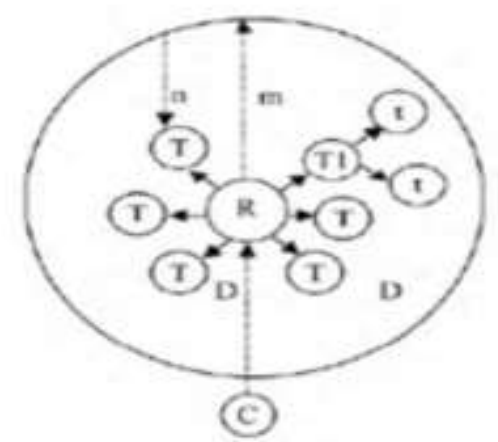

Figure. 4 Model of Remedied Cognitive Reference Point (Wang. 2011)

Hub-and-spoke R-T connection type refers to the process that the same cognitive referential point can lead to several target concepts $(T)$, as is shown in Figure.2. From certain reference point, text conceptualizer(C) predicts and estimated the text statement range, and locates the corresponding series of discussion within the text, as the $T$ in Figure.2. In the cognitive 
processing of understanding certain events, the conceptualizers would locate the reference points that radiate in the text with pronoun anaphora, the repetition of core words, and the substitution of synonym or hyponym, and formulate a concept cluster with the mental path in that semantic domain, organize the statement around the radiant concept cluster. The text statements organized accordingly will possess the internal semantic coherence.

Nested chain R-t connection type refers to the process that one reference point $(R)$ leads to one target concept ( $T$ ), and $T$ that functions as the new reference point will lead to several ts with pronoun anaphora, the substitution of hyponym and hypernym. With the repetitious chaining, the reference points in the previous sentence or the semantic domain of the reference points can provide the potential reference points for the target words in the following sentence. The referential connection between $\mathrm{R}-\mathrm{T}$. $\mathrm{T}$ will provide potential reference points for the $t 1, t 2$ and etc. in the following sentences and construct the referential relation between $\mathrm{T}$ and $\mathrm{t}$. The intertextual sentences with this kind of nested chain network also have coherence. The nested chain $\mathrm{R}$ - $\mathrm{t}$ can explain the application of pronoun and the cognitive foundation of constructing text coherence.

\subsection{Research design}

To specifically account for the application of CTM cognitive framework to construe and generate the text coherence, and to demonstrate the explanatory effectiveness of the CTM framework, this paper selects 30 Abstracts published between Dec 2018 and Jun 2019, which are written by English native speakers and selected from different research fields from Nature official website (https://www.nature.com). This research applies Coh-metrix 3.0, the online analysis software developed for automatic analysis of text coherence by McNamara from University of Memphis, to conduct online automatic analysis to the selected 30 Abstracts. Co-Metrix makes reasonable use of various natural language analyzing techniques such as computational linguistics, corpus linguistics and information retrieval. It can effectively measure the coherence of texts, especially the popularized scientific and academic texts. Coh-Metrix contains a number of factors like cohesive devices and referential cohesion, which affect the coherence of the text. These factors analyze the text coherence from the overlapping phenomenon from the aspects of word, clause, sentence and even paragraph. In the process of variable extraction, Coh-Metrix makes use of the Latent Semantic Analysis (LSA) in the field of computational linguistics. As a new technique in information retrieval, LSA performs in word frequency matrix singular value decomposition, data simplification and compression and reconstruct the matrix. The semantic similarity of linguistic units can be obtained by comparing the vectors that represent the linguistic units like sentences, paragraphs and texts and by computing the cosines between the two vectors. (Landauer et al.1998). Independent of the surface features of the text, LSA can effectively analyze the coherence of the text by analyzing the semantic relatedness of linguistic units within the text (Vollmer2002:15-19). Many scholars measure textual coherence on Coh-Metrix for different research purposes. McCarthy examined the text coherence through latent semantic analysis by identifying the different chapters like Introduction, Methods, Results and Discussion in academic papers, and studied the influence of text coherence on reading comprehension (McCarthy2007). Besides, a small number of studies analyzed the cohesion of the reading comprehension texts in the exam by using the indexes of text coherence of Coh-Metrix (Green2010).

This paper selects the two factors of measuring text cohesion and coherence: LSA and Referential Cohesion (RC) as the referential factors. The materials include the 10 Abstracts of scientific papers that are featured with lower RC and higher LSA selected from 30 Abstracts of scientific papers (Abstract Profiles are shown in Table.1). The specific comparison results are shown in Table.1 and Table.2. Based on the above two factors, the abstract with the highest LSA and the lowest RC was selected for the following case study. The Abstract, with its title The Distribution and Trajectory of Tidal Flats, was published on the official website of Nature on Dec 19, 2018 (https://www.nature.com/articles/s41586-018-0805-8). From the perspectives of hub-and-spoke R-T and nested chain R-T connection types, this paper aims to explore and construct the referential framework of construing and generating coherence, clarity and fluidity of the scientific texts with a rigid logic to describe the scientific facts. We also intend to investigate how the text recipients, while reading the scientific texts with relatively lower RC, dynamically construe and generate the internal coherence of the scientific texts with embodiment.

Table.1. The mean values of LSA and RC from the 10 Abstracts

Title

\begin{tabular}{lll} 
Time & $\begin{array}{l}\text { The } \\
\text { Mean } \\
\text { Value } \\
\text { of } \\
\text { LSA }\end{array}$ & $\begin{array}{l}\text { The } \\
\text { Mean } \\
\text { Value } \\
\text { of RC }\end{array}$ \\
\hline 2018.12 & 0.35 & 0.316 \\
\hline
\end{tabular}




\begin{tabular}{|c|c|c|c|c|}
\hline 2 & A direct test of density wave theory in a grand-design spiral galaxy & 2018.12 & 0.321 & 0.505 \\
\hline 3 & $\begin{array}{l}\text { Long-term experimental hybridisation results in the evolution of a new sex } \\
\text { chromosome in swordtail fish }\end{array}$ & 2018.12 & 0.323 & 0.494 \\
\hline 4 & Hexing the discipline: against the reproduction of continental philosophy of religion & 2019.1 & 0.279 & 0.489 \\
\hline 5 & The ecological drivers of variation in global language diversity & 2019.3 & 0.341 & 0.490 \\
\hline 6 & $\begin{array}{l}\text { Infants' advances in speech perception shape their earliest links between language and } \\
\text { cognition }\end{array}$ & 2019.3 & 0.334 & 0.510 \\
\hline 7 & $\begin{array}{l}\text { Integration of bulk materials with two-dimensional materials for physical coupling and } \\
\text { applications }\end{array}$ & 2019.5 & 0.321 & 0.505 \\
\hline 8 & $\begin{array}{l}\text { High-throughput prediction of the ground-state collinear magnetic order of inorganic } \\
\text { materials using Density Functional Theory }\end{array}$ & 2019.6 & 0.312 & 0.498 \\
\hline 9 & $\begin{array}{l}\text { Cultural variation between neighboring communities of chimpanzees at Gombe, } \\
\text { Tanzania }\end{array}$ & 2019.6 & .281 & 0.508 \\
\hline 10 & $\begin{array}{l}\text { Building in vitro transcriptional regulatory networks by successively integrating } \\
\text { multiple functional circuit modules }\end{array}$ & 2019.6 & $.288^{0}$ & 0.445 \\
\hline
\end{tabular}

Table. 2. The mean values of individual reference indexes under LSA and RC from the 10 Abstracts

\begin{tabular}{|c|c|c|c|c|c|c|c|c|c|c|c|}
\hline & & Abs.1 & Abs. 2 & Abs.3 & Abs.4 & Abs. 5 & Abs.6 & Abs.7 & Abs.8 & Abs.9 & Abs.10 \\
\hline $\mathrm{RC}$ & $\begin{array}{l}\text { The mean values of } \\
\text { overlapping word } \\
\text { stems in all } \\
\text { sentences }\end{array}$ & 0.311 & 0.499 & 0.499 & 0.477 & 0.478 & 0.509 & 0.497 & 0.501 & 0.510 & 0.423 \\
\hline \multirow[t]{3}{*}{ LSA } & $\begin{array}{l}\text { The mean values of } \\
\text { semantic similarity } \\
\text { in all sentences }\end{array}$ & 0.412 & 0.343 & 0.329 & 0.297 & 0.369 & 0.355 & 0.312 & 0.312 & 0.289 & 0.314 \\
\hline & $\begin{array}{l}\text { The mean values of } \\
\text { semantic similarity } \\
\text { of adjacent } \\
\text { sentences }\end{array}$ & 0.288 & 0.299 & 0.322 & 0.260 & 0.312 & 0.313 & 0.331 & 0.311 & 0.273 & 0.262 \\
\hline & $\begin{array}{l}\text { The standard } \\
\text { deviations of } \\
\text { phonetic similarity } \\
\text { in all sentences }\end{array}$ & 0.160 & 0.151 & 0.144 & 0.147 & 0.161 & 0.158 & 0.142 & 0.141 & 0.150 & 0.161 \\
\hline
\end{tabular}




\section{The case study}

The CTM that this paper attempts to analyze the text coherence from a macro perspective. This framework has universal linguistic representation and is the conceptual prototype of many linguistic constructions. The referential points of a text determine the writers' psychological processing when they try to organize the text layout. The cognitive referential points rely on the unity of referential points to interpret the local and global coherence.

The title of a scientific text is a high generalization of the text content an accurate reflection of the researcher's writing intention. Harmon\& Gross (2009) proposed that titles play two roles in predicting text content and text comprehension. One role is to guide the existing cognitive structure of the discourse construers, form the mental direction and construct the new cognitive structure. Therefore, the title has the first function of text guidance. Another role is to navigate the construers to initially form the basic cognitive framework related to text content and thought. The predictive functions of titles can stimulate the thinking schema of text construers in corresponding field, associative the cognition structure of text construers and form a new knowledge system. Therefore, the analytical points of The Global Distribution and Trajectory of Tidal Flats are started from the title and used to extract the cognitive schema related to text construers and to activate the relevant semantic domain in the brain.

\subsection{The dynamic analysis of the case Abstract}

To regard the title of The Global Distribution and Trajectory of Tidal Flats as the starting points of text coherence can help the text recipient to predict the content of the paper, initiate the text construal, extract the fundamental cognitive framework that generates the semantic domain and activate the related conceptual system associated with long term memory and working memory.

According to the logic flow, the case Abstract can be divided into three semantic chunks. The first semantic chunk includes the first two sentences. Here is the detailed information: "Increasing human populations(R) around the global coastline have caused ( $\mathrm{T}$ ) extensive loss ( $\mathrm{t} 1$ ), degradation ( $\mathrm{t} 2$ ) and fragmentation of coastal ecosystems ( $\mathrm{t} 3$ ), threatening $(\mathrm{T})$ the delivery of important ecosystem services. As a result, alarming losses (T) of mangrove, coral reef, seagrass, kelp forest and coastal marsh ecosystems have occurred." The hub-and-spoke R-T connection type and nested chain R-t connection type would be used to construe and generate the Abstract coherence. The initial cognitive referential point (R) is "increasing population". Based the SVO syntactic structure, the first targeted point ( $\mathrm{T}$ ) is "have caused", and the sequentially nest-chained three objects "loss(t1), degradation(t2), fragmentation(t3)". With the linear propulsion of the Abstract text, the second targeted point $(T)$ that shares the same level as "have caused" can be ascertained. Hence the local coherence of this sentence can be achieved with the visually initial referential point "increasing human population" and two peer target points "have caused" and "threatening". The logic continuity between the first sentence and the second sentence is realized by the cohesive phrase "as a result". First of all, the semantic coherence is internally echoed by the referential point"alarming losses"and "caused" "threatening" in the first sentence. Then the substitution of "coastal ecosystem" with its corresponding hyponym "mangrove, coral reef, seagrass" actualizes the latent semantic relevance between the first sentence and the second one. The dynamic cognitive activity of the sentences under the above semantic chunk can be shown in Figure.3.

The second semantic chunk refers to the third sentence of the Abstract: "However, owing to the difficulty of mapping intertidal areas globally, the distribution and status of tidal flats-one of the most extensive coastal ecosystems-remain unknown". Its cognitive referential point is the cohesive word "however" (R). The following prepositional phrase "owing to" can cognitively assist the viewers in determining the function of this part, and sequentially their attention will flow to the targeted concept (T): "distribution and status of tidal flats", of which the semantic domain (D) can be framed as "tidal flats". The CRP construal framework from Langacker, the cohesive devices, prepositional phrases, and apposition structure work together to place this semantic chunk as the transitional part in the whole Abstract. The second semantic chunk integrates the first and the third semantic chunk and thus enhances the global coherence of this Abstract.

The third semantic chunk covers sentences four to eight: "Here we present an analysis .... We find that.... About $70 \%$ of the global extent of tidal flats is found in three continents.... For regions...-we estimate that ...tidal flats were lost .... Extensive degradation... increased coastal erosion and sea-level rise signal a continuing negative trajectory .... Our ... dataset delivers ....". This coherence of this part can be construed and generated with hub-and-spoke R-T connection type and nested chain R-t connection type. The cognitive referential point is "here", and the sequentially activated target concept is the second personal pronoun "we". In this semantic chunk, various formal substitutions of the second personal pronoun have been implemented. For example, the second personal pronoun "we" is replaced with the second personal possessive pronoun "our". Consequently, the four related target points "we present", "we find", "we estimate", "our ...delivers" can be chained, of which the second personal pronoun "we" plays the leading thematic function and cognitively generates the local coherence of the Abstract in this semantic 
chunk. Within this framework, every target point $(T)$ is nest-chained with a t. The dynamic cognitive referential activity is shown in Figure.4.

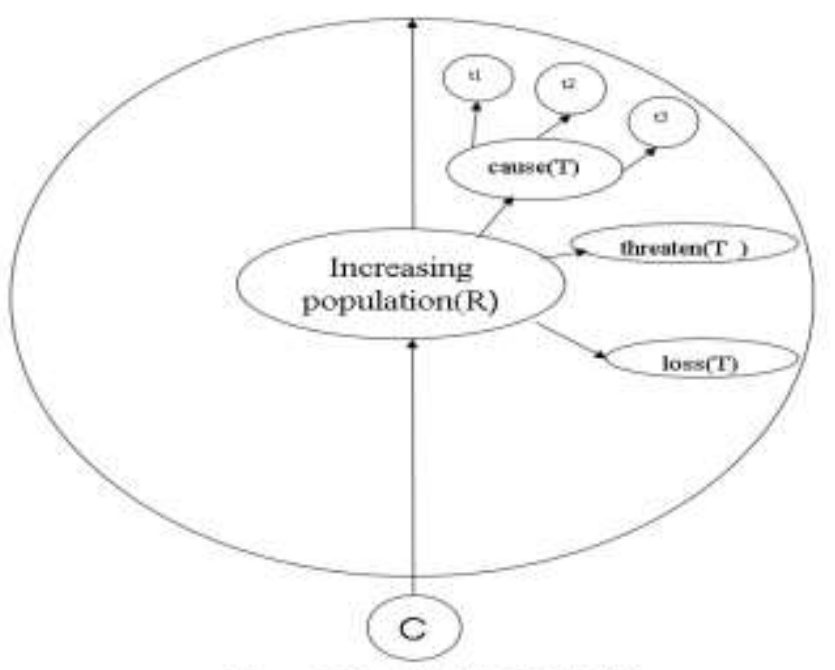

Figure. 3 Nested chain R-t RCRP

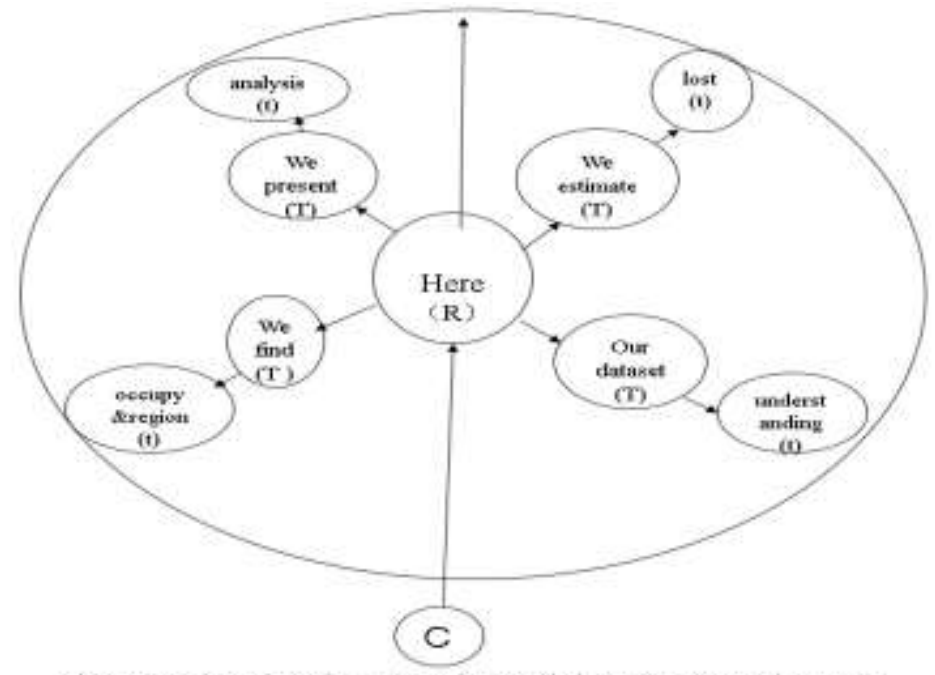

Figure. 4 Hub-and-Spoke R-T and Nested chain R-t comection types

Integrating the dynamic cognitive activity shown in Fig.3 and Fig.4 from RCRP, the text composers and recipients generate and construe the mental simulation of the first semantic chunk, extract the related background information to tidal flats, and insert the information to the text comprehension. The second semantic chunk uses the cohesive word "however" to put forward the research question, predict and construct the guiding connection to the third semantic chunk. With the cognitive reference point "here" and the sequential second personal pronouns "we" and "our", the third semantic chunk contributes to integrating the psychological cognitive representation of the text recipients, namely the contributing achievements of the text composers.

Overall, in this case Abstract, the synonymic substitution of the core words, the hyponymic and hypernymy substitution of the core

words, the pronoun anaphora and cohesive words together realize bridging process between the nest-chained $t$ and target concepts. After generating the cognitive schema, text construers can, based on their research need, decide which semantic part is worthwhile more attention.

As shown in Fg.1, Fig.2, Fig.3, and Fig.4, if text composer or construer, while comprehending and generating the Abstract coherence of scientific paper, can mobilize different modal inputs like visual (text, video, pictures, table, figure, chart) and motor (drawing mind map) situational input, the cognitive load of the composers and construers can be reduced and the memory length and depth can be improved. 


\section{Conclusion}

Integrating Langacker's model of Cognitive Reference Point (CRP) and Wang Yin's model of Remedied Cognitive Reference Point (RCRP), the cognitive comprehending framework of CTM is employed in this study to test, construe and generate the coherence of scientific texts. This study has demonstrated the efficacy of the CTM framework, consisting of cognitive reference point, target concept point and the processing mental path (CTM), to assist the dynamic construal and generation of local and global coherence in Abstracts of scientific papers. It establishes a psychological and cognitive referential framework for Abstract viewers, which enables viewers to efficiently construe and construct the local and global coherence of Abstracts in a more dynamic and situational way. With the guidance of the framework, scientific text composers and construers could efficiently extract the key information by reconnecting previously possessed information, and reconstructing the new information framework. However, the research results are tested only within limited number of Abstracts in scientific articles. In the future, the efficacy of CTM could be further verified with the assistance of psychological experiments and the corpus containing scientific texts.

Funding: This research was funded by China Ministry of Education Humanities and Social Sciences Research Project, grant number 18YJCZH113.

Acknowledgments: Thanks are due to Yimin Huang for assistance with the materials collection and to Jing Yin for valuable discussion.

Conflicts of Interest: The author declares no conflict of interest. The funders had no role in the design of the study; in the collection, analyses, or interpretation of data; in the writing of the manuscript, or in the decision to publish the results.

\section{References}

[1] Barsalou, L.W., Simmons W.K., Barbey, A.K., \& Wilson, C.D. (2003). Grounding conceptual knowledge in modality-specific systems, Trends in Cognitive Scinece, (7), 84-91.

[2] Beaugrande, R.D., \& Dressler, W. (1981) Introduction to Text Linguistics. Longman, 23-26.

[3] Chiel H, Beer R. (1997). The brain has a body: Adaptive behavior emerges from interactions of nervous system, body and environment. Trends Neurosci, (20), 53-557.

[4] Clark, A., (1997). Being there: Putting Brain, Body and World Together Again, Philosophy, (10), 23-26.

[5] Gernsbacher, Morton Ann. (1992) Language Comprehension as Structure Building, Psycoloquy, 1-5.

[6]Givon.T.(1990). Syntax: A Functional-Typological Introduction, Vol.2. John Benjamins, 911-914.

[7] Givón, T. (1993). Coherence in Text, Coherence in Mind, Pragmatics \& Cognition 1(2), 171-227.

[8] Glenberg, A.M.\& Robertson, D. A. (1991). Indexical understanding of instructions., Discourse Processes, 1-26.

[9] Glenberg, A.M., \&Gallese, V. (2011). Action-based language: A theory of language acquisition, comprehension, and production. Cortex, (4), 110.

[10] Green, A., unaldi, A., \&Weir,C.(2010).Empiricism versus connoisseurship: Establishing the appropriacy of texts in tests of academic reading. Language Testing, (3), 1-21.

[11] Harmon, J., \& Gross, A. (2009). The Structure of Scientific Titles, Journal of Technical Writing and Communication, 39(4), 455-465.

[12] Hesselbach, A, Petering, D.A, Berg, C.A, Tomasiewicz, H \& Weber, D. (2012). A guide to writing a scientific paper: a focus on high school through graduate level student research, Zebrafish, 69-74.

[13] Jie Yang, Hua Shu, Yanchao Bi, Youyi Liu, \&Xiaoyi Wang, (2011). Dissociation and association of the embodied representation of tool-use verbs and hand verbs: An fMRI study, Brain and Language, 119(3), 167-74.

[14] Koulaidis,V, Dimopoulos, K, \& Sklaveniti, S. (2001). Analyzing the Texts of Science and Technology: School Science Textbooks and Daily Press Articles in the Public Domain, Learning for the Future Proceedings of the Learning Conference, 4-25.

[15] Langacker. R.W. (2001). Discourse in cognitive grammar. Cognitive Linguistics, (12), 23-26.

[16] Langacker.R.W.(2004). Grammar and Conceptualization. Mounton de Gruyter, 13-20.

[17] Landauer, T. K, P.W.Foltz\&D.Laham.(1998). Introduction to latent semantic analysis. Psychological Review (8), 363-394.

[18]McCarthy.P.,Briner,S.,Rus,V.,\&McNamara,D.(2007).Textualsignatures:Identifying text types using latent semantic analysis to measure the cohesion of text structures. Springer.

[19] Norman, K. A., Polyn, S. M., Detre, G. J., \& Haxby, J. V. (2006). Beyond mind-reading:Multi-voxel pattern analysis of fMRI data. Trends in Cognitive Sciences, 10(9), 424-430.

[20] van Dijk,T.A. (2004). From text grammar to critical discourse analysis: A brief academic autobiography, 10-15.

[21] van Dijk, T.A.(1980). Microstructure: An Interdisciplinary Study of Global Structures in Discourse, Interaction and Cognition. Lawrence Erlbaum Associates, 25-28.

[22] Shakiba*, A, Saif, A. A, Asadzadeh, H, \& Ghavam, S. E, (2013) Investigating the effect of local and global cohesion on improving reading comprehension of students with low and high prior knowledge, European Journal of Experimental Biology, 3(2), 551-555.

[23] Vollmer,H.(2002).Textual Coherence: Basics of Text linguistics. University of Osnabruck.

[24] Chen, Qilong, (2001). Cognitive interpretation of discourse formal and semantic connection. Journal of PLA University of Foreign Languages , (1), 23-29.

[25] Wang, Yin. (2003). Cognitive linguistics and discourse analysis: the analytical perspectives of Langacker, Foreign Language Teaching and Research, (3), 83-88. 
[26] Wang, Yin. (2005). The cognitive analysis of discourse coherence: the interpretation of discourse coherence from embodied philosophy and cognitive linguistics. Foreign Language Research. (4), 16-22.

[27] Wang, Yin. (2011) The remedied model of cognitive reference points and discourse coherence construction. Foreign Language and Foreign Language Teaching, 6-10.

[28] Weidemann, C.T, Kragel, J.E, Lega, B, \&Kahana, M.J. (2019). Neural activity reveals interactions between episodic and semantic memory systems during retrieval, Journal of Experimental Psychology General, 148(1), 1-12.

[29] Wilson, Margaret. (2002). Six views of embodied cognition, Psychonomic Bulletin \& Review, 9(4), 625-636.

[30] Zhang, Delu, Liu, Rushan. (2003). The development and application of discourse coherence and cohesive theory. Shanghai Foreign Language Education Press.

\section{Appendix 1}

\section{Abstract transcript of the case study}

\section{The global distribution and trajectory of tidal flats}

Increasing human populations around the global coastline have caused extensive loss, degradation and fragmentation of coastal ecosystems, threatening the delivery of important ecosystem services. As a result, alarming losses of mangrove, coral reef, seagrass, kelp forest and coastal marsh ecosystems have occurred1,2,3,4,5,6. However, owing to the difficulty of mapping intertidal areas globally, the distribution and status of tidal flats-one of the most extensive coastal ecosystems-remain unknown7. Here we present an analysis of over 700,000 satellite images that maps the global extent of and change in tidal flats over the course of 33 years (1984-2016). We find that tidal flats, defined as sand, rock or mud flats that undergo regular tidal inundation7, occupy at least $127,921 \mathrm{~km} 2(124,286-131,821 \mathrm{~km} 2,95 \%$ confidence interval). About $70 \%$ of the global extent of tidal flats is found in three continents (Asia (44\% of total), North America (15.5\% of total) and South America (11\% of total)), with $49.2 \%$ being concentrated in just eight countries (Indonesia, China, Australia, the United States, Canada, India, Brazil and Myanmar). For regions with sufficient data to develop a consistent multi-decadal time series-which included East Asia, the Middle East and North America-we estimate that 16.02\% (15.62-16.47\%, 95\% confidence interval) of tidal flats were lost between 1984 and 2016. Extensive degradation from coastal development1, reduced sediment delivery from major rivers8,9, sinking of riverine deltas8,10, increased coastal erosion and sea-level rise 11 signal a continuing negative trajectory for tidal flat ecosystems around the world. Our high-spatial-resolution dataset delivers global maps of tidal flats, which substantially advances our understanding of the distribution, trajectory and status of these poorly known coastal ecosystems. (272 words). 